\title{
The (cost) effectiveness of guided internet- based self-help CBT for dialysis patients with symptoms of depression: study protocol of a randomised controlled trial
}

Els Nadort ${ }^{1,2,3^{*}}$ (D), Robbert W. Schouten ${ }^{1,2,3}$, Friedo W. Dekker ${ }^{4}$, Adriaan Honig ${ }^{2,3}$, Patricia van Oppen ${ }^{3,5}$ and Carl E. H. Siegert ${ }^{1}$

\begin{abstract}
Background: Only a minority of dialysis patients with depressive symptoms are diagnosed and receive treatment. Depressive symptoms are highly prevalent in this population and are associated with adverse clinical outcomes. Underlying factors for this undertreatment may be the lack of evidence for the safety and effectivity of antidepressant medication, the reluctance of patients to adhere to antidepressant medication, the lack of mental healthcare provision in somatic healthcare environments and end-stage renal disease (ESRD) related physical limitations that complicate face-to-face psychotherapy. Guided Internet-based self-help treatment has demonstrated to be effective for depressive symptoms in other chronic patient populations and may overcome these barriers. The aim of this study is to investigate the (cost) effectiveness of a guided Internet-based self-help intervention for symptoms of depression in dialysis patients.

Methods: This study is a cluster randomized controlled trial (RCT) that investigates the effectiveness of a 5-week Internet-based self-help Problem Solving Therapy (PST) for depressive symptoms in dialysis patients. Depressive symptoms will be measured using the Beck Depression Inventory - second edition (BDI-II), with a cut-off score of $\geq 10$. We aim to include 206 dialysis patients with depressive symptoms who will be cluster randomized to the intervention or the Care as Usual (CAU) control group. Secondary outcomes will include anxiety symptoms, quality of life, economic costs and clinical outcomes, such as inflammatory factors and hair cortisol levels. Assessments will take place at baseline (T0), 2 weeks after intervention (T1) and 6 months (T2), 12 months (T3) and 18 months (T4) after intervention. The control group will be measured at the same time points. Analysis will be based on the intention-to-treat principle. Mixed models will be used to assess the changes within each condition between pretreatment and post-treatment.
\end{abstract}

Discussion: If demonstrated to be (cost) effective, Internet-based PST will offer new possibilities to treat dialysis patients with depressive symptoms and to improve their quality of care.

Trial registration: Dutch Trial Register: Trial NL6648 (NTR6834) (prospectively registered 13th November 2017).

Keywords: Depression, Dialysis, Internet, Self-help intervention, EHealth, CBT, PST, RCT, Cost-effectiveness, Hair cortisol

\footnotetext{
* Correspondence: e.nadort@olvg.nl

'Department of Nephrology, OLVG hospital, Jan Tooropstraat 164, 1061 AE

Amsterdam, Netherlands

2Department of Psychiatry, OLVG, Jan Tooropstraat 164, 1061 AE Amsterdam,

Netherlands

Full list of author information is available at the end of the article
}

(c) The Author(s). 2019 Open Access This article is distributed under the terms of the Creative Commons Attribution 4.0 International License (http://creativecommons.org/licenses/by/4.0/), which permits unrestricted use, distribution, and reproduction in any medium, provided you give appropriate credit to the original author(s) and the source, provide a link to the Creative Commons license, and indicate if changes were made. The Creative Commons Public Domain Dedication waiver (http://creativecommons.org/publicdomain/zero/1.0/) applies to the data made available in this article, unless otherwise stated. 


\section{Background}

Only in a minority of dialysis patients, depressive symptoms are diagnosed and treated [1]. However, depressive symptoms are highly prevalent in this population and are associated with adverse clinical outcomes [2]. These symptoms are a major burden to the individual dialysis patient causing decreased quality of life and are associated with decreased adherence to dialysis prescription and lifestyle advice, increased hospitalization and decreased survival $[1,3,4]$. Furthermore, the social and economic costs related to depression in the dialysis population are substantial. The effect of depressive symptoms on the increase of health care costs seem to be independent of other factors, such as comorbidities, dialysis aspects and demographic variables $[1,4]$.

Depressive symptoms and its impairments do not remit spontaneously if left untreated [5, 6]. Underlying factors for under-treatment of depressive symptoms with medication are a lack of evidence for the safety and effectivity of antidepressant medication in dialysis patients $[7,8]$ and the reluctance of patients to adhere to antidepressant medication [9, 10]. Psychotherapy could be a safe alternative, with promising results in the few published trials in the end-stage renal disease (ESRD) population [11-13]. However, barriers to receive psychotherapy are the lack of mental healthcare provision in somatic healthcare environments and ESRD related physical limitations such as fatigue and other physical impairments that may reduce the ability of patients to attend face-to-face psychotherapy.

Guided online self-help cognitive behavioural treatment tailored to dialysis patients may be a promising tool for treatment of depression in this population. A guided cognitive-behavioural internet-based self-help intervention can overcome various barriers with respect to face-to-face interventions as it is easy accessible, home or dialysis-based and can be followed in one's own limited time [14]. Self-help treatment has been proven effective in psychological distress in people with and without chronic physical health conditions [15-19]. These self-help interventions have proven equally effective in terms of reducing depressive symptoms and adherence compared to face-to-face psychological interventions, when they are guided by a therapist [20]. Feasibility trials with online self-help cognitive behavioural treatment in dialysis patients show promising results but, to the best of our knowledge, no adequately powered randomized controlled trial (RCT) has yet been performed [16, 21-24].

A commonly used brief, structured, psychological intervention adapted for use in the medical setting is Problem Solving Therapy (PST). PST is based on the assumption that depressive symptoms are caused by difficulties patients encounter in life. The goals are to teach a structured problem-solving technique to help solve the patients' current problems and to provide a sense of mastery and self-control by providing a positive experience of problem-solving $[25,26]$.

Besides the lack of evidence for the effectiveness of treatment on improving depressive symptoms in dialysis patients, more insight is needed in the possible mechanisms that are involved between somatic markers and depressive symptoms in this patient population. Numerous studies have suggested a parallel inflammatory pathway between depression and ESRD via elevated levels of inflammatory cytokines [27] or a relation between hyperactivity of the hypothalamic-pituitaryadrenal (HPA) axis and depressive symptoms [28]. These pathways are not yet fully understood and are founded on associations found in observational studies. Monitoring changes in stress and inflammatory markers preand post-treatment may provide insight in the direction of the hypothesized causal pathways.

There are no adequately powered studies that have examined the effectiveness of guided Internet-based selfhelp treatment in dialysis patients. Data from applying PST in other medical settings and from smaller feasibility trials in dialysis patients show promising results in improving depressive symptoms. We hypothesize that this intervention will lead to lower depressive symptoms, is better accessible and can be offered at low costs [29, 30]. The aims of this RCT are therefore multiple. First, we will evaluate clinical and cost-effectiveness of a guided internet-based self-help PST for dialysis-patients on the primary outcome measure of depressive symptoms. Secondly, we will examine the effect on the secondary outcome measures anxiety and quality of live. And thirdly, we will examine biochemical mechanisms of depression by investigating changes in inflammatory parameters and hair cortisol levels pre- and posttreatment.

\section{Methods \\ Study design}

This study is a multicentre, cluster RCT with an active intervention arm and a Care as Usual (CAU) control arm. The intervention is a guided 5-week Internet-based self-help PST treatment, offered on tablet-computers during dialysis sessions. The internet-based treatment is based on face-to-face PST and is guided by a therapist, who gives individual feedback to the patients via the online portal. Eligible and consenting patients will be assessed at baseline (T0), within 2 weeks after the intervention (T1), at 6 months (T2), at 12 months (T3) and at 18 months (T4) after intervention. The control group will be assessed at the same time points. Data will be collected by self-reported questionnaires during dialysis sessions. In parallel we will conduct an economic 
evaluation in order to assess cost-effectiveness and monitor changes in stress and inflammatory markers pre- and post-treatment to examine biochemical mechanisms of depression.

The study protocol, information brochure and informed consent were approved by the Medical Ethics Committee of MEC-U, Nieuwegein, the Netherlands (registration number: NL58520.100.17). Protocol modifications will be reported to and approved by the Medical Ethnics Committee before implementation in the trial. Written informed consent is obtained from all participants. Fig. 1 displays the flow diagram of the study design. This protocol is written in accordance with the SPIRIT guidelines.

\section{Recruitment}

Dialysis patients will be recruited from 18 participating dialysis centres in 10 cities in the Netherlands. A list of study sites can be found in Additional file 1. We will approach and inform all eligible dialysis patients in the participating centres about the study in close collaboration with all stakeholders, such as nephrologists, nurses and social workers. The treating nephrologist will inform the patient on the trial and introduces the research assistant in the dialysis centre. If patients are willing to participate, written informed consent will be obtained by the research assistant. The attending nephrologist will be informed about participation. After giving consent, patients will be requested to complete a self-assessment questionnaire (T0) on depressive symptoms, anxiety symptoms, quality of life, dialysis symptoms and several socio-demographic questions. Blood and hair samples will be taken and stored for analysis of inflammatory parameters and cortisol levels.

\section{Trial inclusion criteria}

Chronic, adult dialysis patients, defined as being (i) 18 years or older and (ii) $>90$ days on dialysis treatment, who are (iii) able to fill in a questionnaire in Dutch and have (iii) a depressive symptoms score of 10 or higher on the Beck Depression Inventory - second edition (BDI-II) [31], will be randomized to the intervention or control arm. This cut-off value of 10 showed promising results in earlier feasibility trials $[21,23]$.

\section{Observational cohort inclusion criteria}

Chronic, adult dialysis patients who are excluded from the randomization because of a low score on the BDI-II or patients who have insufficient Dutch language skills, are offered to participate in a parallel observational cohort study. Questionnaires will also be available in Arabic, English and Turkish. In this manner we will gain information on the excluded patients and thus the generalizability of the results, which could aid in the implementation in clinical practice.

\section{Exclusion criteria}

Patients will be excluded if they are actively suicidal. If patients report suicidal ideations on item 9 of the BDI-II "suicidal thoughts and wishes" by scoring '2' ("I would like to kill myself") or ' 3 ' ("I would kill myself if I had the chance"), suicide risk will be further assessed by a study doctor under supervision of a psychiatrist. If the patient is actively suicidal, the patient will be excluded from the study and the attending nephrologist will be informed and advised to refer the patients for adequate safety and treatment.

\section{Randomization}

Cluster randomisation will be applied to reduce possible contamination between both arms of the trial. Participants will be cluster-randomized in a 1:1 allocation to the intervention or CAU after baseline measurements (T0). Clusters will be based on the shift of the dialysis session per dialysis centre. The average dialysis centre has 4 major shifts: Monday morning, Monday afternoon, Tuesday morning and Tuesday afternoon. A total of 72 clusters of average 3 patients in 18 participating dialysis centres will be present in our study. Clusters will be randomized using stratified blocks per participating dialysis centre.

Randomization will be performed and registered by an automated computer software programme to ensure independent allocation. Baseline measurements will be completed prior to randomization. The coordinating researcher assigns the intervention to participants. Although the treating nephrologist will not be actively informed about the depression score and allocation, we do not consider them blinded as patients and investigators will not be blinded. Outcome assessors are blinded and data analysts will be blinded until primary analysis will be performed.

\section{Intervention}

The internet-based self-help intervention examined in this study is an online psychotherapy, based on PST principles [26]. PST focusses on developing coping skills and is concentrated on practical problems which people face in their daily lives. An existing evidence based internet version of PST, which has proven to be effective in similar somatically ill patient populations, [30], was adjusted for use in the dialysis population, while conserving the intent of the original PST-based intervention. Modifications concerned additional information about dialysis treatment and its psychosocial consequences, real-life examples from dialysis patient focus groups and transforming the written information into easily 


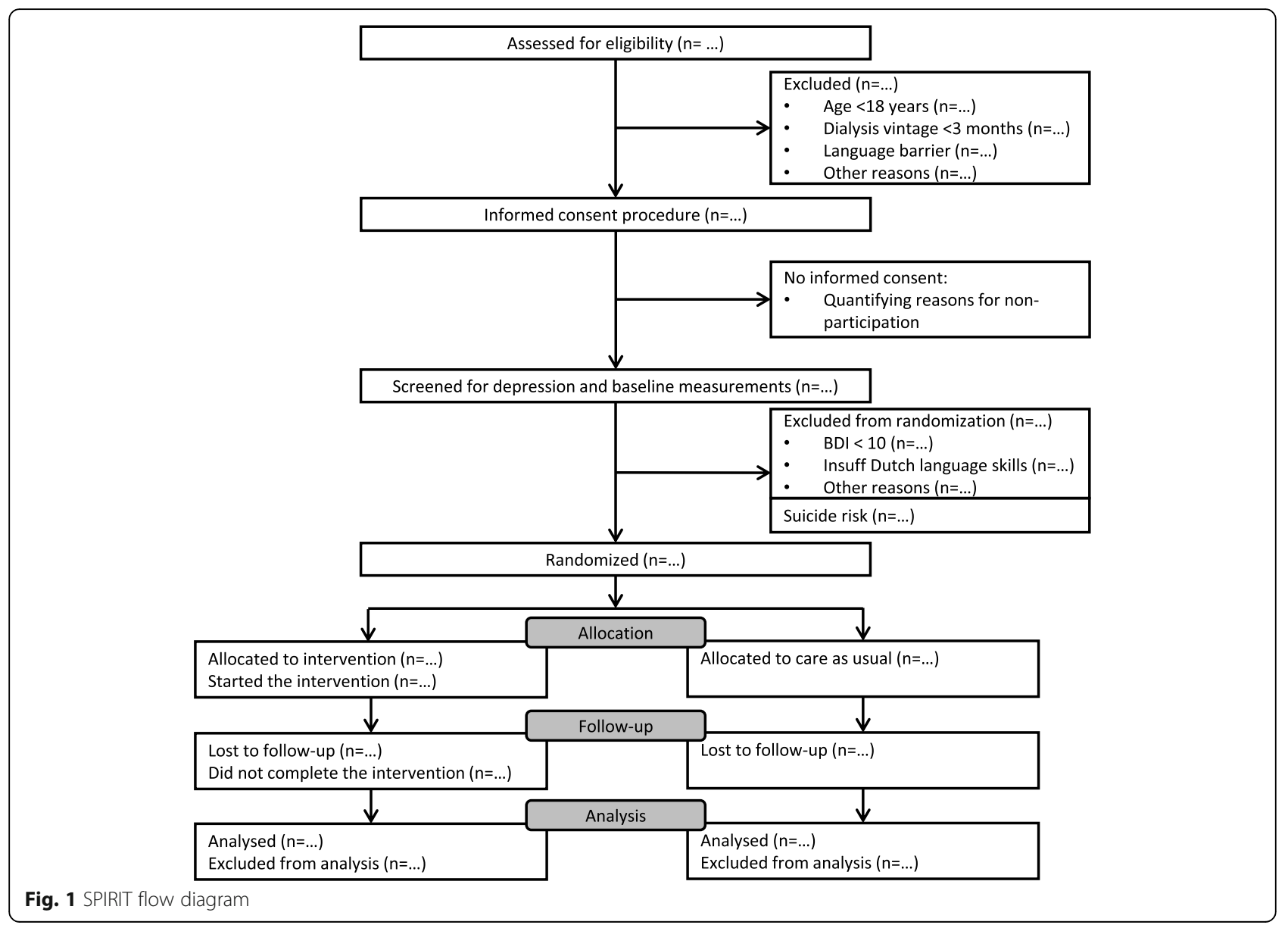

understandable animations. The text and animations were rewritten to comply with a 'B1' language reference level [32].

The intervention consists of five modules with explanatory text, animations, figures and exercises and is called 'Worry Less for Dialysis Patients' (in Dutch: "Minder Zorgen voor Dialyse Patiënten"). Patients are requested to complete 1 session each week and to finish the module within 6 weeks. However, there will be a possibility to extend this period up to 10 weeks, which will be documented. Patients are offered to complete the sessions on a tablet computer provided by this trial during dialysis sessions, but if preferred it can also be done from home on a private tablet or computer. If patients are unfamiliar with tablet computers, they are offered the opportunity to receive a printed booklet of the intervention. If patients have problems with the use of the tablet due to unfamiliarity, physical limitations, shunt use in the dominant arm of Dutch writing problems, they will be supported by a member of the research team, which will be documented. Supported care within the module is provided by a trained therapist and consists of weekly online feedback on their assignments.
Patients have the possibility to request their therapist for additional support via the website. Treatment nonadherence and drop-outs will be discussed with the patients. Reasons for not completing the modules and patient satisfaction will be obtained via a short evaluation questionnaire. If a patient expresses suicidal ideations in the assignments of the intervention, suicide risk will be assessed over telephone by the supporting therapist under supervision of a psychiatrist. If the patient is actively suicidal, the patient will be excluded from the study and the attending nephrologist will be informed and advised to refer the patient for adequate safety and treatment.

\section{Control}

Patients randomized to the control group do not receive Internet-based PST during standard haemodialysis treatment. Both patients in the intervention and in the control group are free to accept any medical or psychological intervention during the study (CAU). The received mental healthcare will be monitored through electronic patient records and self-reported healthcare utilization. 


\section{Feasibility}

In 2016 we conducted a feasibility test with 15 patients from different age categories in the dialysis clinic of the OLVG-West hospital in Amsterdam. Every patient was given a tablet computer with one of the modules of the PST intervention. Patients evaluated the course with a grade of 7 out of 10 . The instruction and lay-out was clear for most of the patients, respectively 75 and $90 \%$. The introduction and several explanatory texts were adjusted according to suggestions from participants in the feasibility test and patient focus groups.

Initially we used BDI $>13$ as inclusion criteria. Due to new available research in feasibility trials we amended the protocol to BDI $>10$ which has been approved by the Medical Ethics Committee MEC-U [21, 23].

\section{Outcome measures}

\section{Patient characteristics}

Demographic self-reported items in the questionnaire will include postal code, marital status, number of children, education, profession, ethnicity, payed labour hours, smoking, alcohol usage and psychiatric disorders in the family. Data extracted from patient files will include gender, age, dialysis vintage, vascular entrance, registration on transplantation list, somatic and psychiatric comorbidities according to Davies Comorbidity Index, body mass index (BMI), primary cause of kidney failure, medication, routine laboratory measurements and change in dialysis modality. Psychotherapy and medication usage will be registered between follow-ups. The self-reported measurements will be conducted with printed self-reported questionnaires handed out during the dialysis session of the participants. Table 1 describes the measures used at each assessment point.

\section{Primary outcomes}

Depressive symptoms are assessed using a selfquestionnaire, the BDI-II [33, 34]. Respondents are asked to rate how much each of these symptoms bothered them in the past week, on a scale ranging from 0 (not at all) tot 3 (severely). The total score has a minimum of 0 and a maximum of 63 . Treatment response will be based on the change in depressive symptoms defined by a change in the sum score of the BDI-II. The BDI-II has been validated and extensively used in the dialysis setting [2, 31, 35]. Furthermore, the Quick Inventory of Depressive Symptomatology (QIDS-SR16) self-report questionnaire will be used to assess the specific depressive symptoms, its severity and its symptom domains [36].

\section{Secondary outcomes}

Secondary outcome assessment based on self-report will include measures of anxiety, quality of life, health care utilization and the prevalence, severity and impact of symptoms in dialysis patients. Anxiety will be assessed with the Beck Anxiety Inventory (BAI) [37]. Quality of life will be measured using the Short Form-12 (SF-12). The 12-item Short Form Health Survey was developed for patients with chronic conditions. The SF-12 has been validated and frequently used in the dialysis patient groups [38]. Prevalence, severity and impact of symptoms of dialysis will be assessed with the Dialysis Symptom Index (DSI) [39]. Clinical outcomes include mortality and hospitalization. Mortality is measured using the European Renal Association - European Dialysis and Transplant Association (ERA-EDTA) coding system to make differences between cardiovascular and non-cardiovascular mortality. Hospitalization is

Table 1 Summary of measures

\begin{tabular}{|c|c|c|c|c|}
\hline Measure & T0: baseline & T1: 2 weeks posttreatment & T2: 6 months posttreatment & T3-T4: 12-18 months posttreatment \\
\hline \multicolumn{5}{|l|}{ Self-report measures } \\
\hline Demographics & $x$ & & & \\
\hline $\mathrm{BDI}-\mathrm{II}$ & $x$ & $x$ & $x$ & $x$ \\
\hline BAl & $x$ & $x$ & $x$ & $x$ \\
\hline QIDS-SR16 & $x$ & $x$ & $x$ & $x$ \\
\hline SF-12 & $x$ & $x$ & $x$ & $x$ \\
\hline EQ-5D & $x$ & $x$ & $x$ & $x$ \\
\hline DSI & $x$ & $x$ & $x$ & $x$ \\
\hline Short Tic-P & $x$ & $x$ & $x$ & $x$ \\
\hline Hair questionnaire & $x$ & $x$ & $x$ & \\
\hline Evaluation of intervention & & $x$ & & \\
\hline Data extraction from patients files & $x$ & $x$ & $x$ & $x$ \\
\hline Biochemical parameters & $x$ & $x$ & $x$ & $x$ \\
\hline Hair cortisol & $x$ & $x$ & $x$ & \\
\hline
\end{tabular}


defined as the number and reason of hospital admissions from baseline till end of the study period.

\section{Economic evaluation}

A validated health-related quality of life instrument will be used to assess quality-adjusted life years (QALY) health gains. For this purpose we will use the Dutch version of the 5 level EuroQol five dimension scale (EQ$5 \mathrm{D})$, a generic quality of life instrument which comprises of five domains: mobility, self-care, usual activities, pain/ discomfort and anxiety/depression. The EQ-5D index is obtained by applying predetermined tariffs (utility weights) to the five domains. The Dutch tariffs of the EQ-5D will be used for computing the QALYs [40]. This index provides a societal-based global quantification of the patient's health status. Furthermore, the EQ-5D will be compared with the SF-12 health related quality of health questionnaire, which is used often in patients on chronic dialysis therapy. Healthcare usage will be measured using part one of the Tic-P self-report together with data from patient files [41].

\section{Inflammatory factors and cortisol}

Besides data on biochemical parameters extracted from patient files, blood samples will be taken to measure cytokines interleukin 1-beta (IL-1B), IL-6, IL-10, high sensitivity C-reactive protein (Hs-CRP) and tumour necrosis factor alpha (TNFa). Peripheral blood before dialysis will be collected in anticoagulant-free EDTA and serum tubes. All samples will be immediately centrifuged at $1200 \mathrm{~g}$ for $10 \mathrm{~min}$ and stored in aliquots at $-80^{\circ} \mathrm{C}$ until analysis. Hair samples will be taken from the back of the head as close as possible to the scalp to measure mean cortisol concentrations from the past 3 months [42].

\section{Data management and monitoring}

Patient flow in the participating centres will be organised using a secured tailor-made Access database. The decoding list will be kept in the Investigator Site File in a secured place in the dialysis department. Data-entry will be coded and entered in Castor [43]. Range limitations will be build into Castor to prevent misclassification and measurement error. Research assistants will be trained in data collection and data entry to enhance data quality.

The risk of this trial is classified as 'negligible' and a study specific monitoring plan is created in close corporation with the data monitoring committee which includes double data entry checks. If deemed necessary by the monitoring committee, monitoring can be intensified. No adverse events are to be expected specifically related to this intervention. Actively suicidal participants will be excluded from the trial as described above.

\section{Sample size}

The power calculation is based on the comparison between $\mathrm{T} 1$ to T0 between the intervention and the control group. We took the conservative small to medium effect size (Cohen's $\mathrm{d}=0.4$ ) on the primary outcome measure, while using a power 0.80 , with alpha set on 05 . Therefore, a total set of $N=99$ patients is required in each arm. The design effect of cluster-randomization is estimated to be 1.04. After adjustment for cluster randomization, sample size is calculated to be $N=206$ in total (103 per arm).

\section{Statistical analysis}

We will quantify the flow of participants through the study using frequencies and percentages in accordance with the SPIRIT flow diagram shown in Fig. 1. Missing data will be reported and discussed in the manuscripts on the trial. Depending on the type of missing data, multiple imputation will be used to handle missing data. Descriptive statistics will be used to describe non-consent, treatment adherence and completion, drop-out and exclusion. The main analysis to assess the effectiveness of the intervention will test differences in the change in depression scores pre-and post-treatment between the intervention and control arms. Analysis will be done per intention to treat principle. Differences in change in depressive symptoms and other continuous secondary outcomes between intervention and control will be assessed using mixed models with the respective clusters, centres and baseline scores as covariates. The analyst will be blinded to the treatment group allocation. Treatment effect over time will be tested by adding a group*time interaction term into the model. Regression models will be used to explore (biochemical) mechanisms. Multivariable adjustment will be done deliberately within the causal pathway in order to explain potential mechanisms.

\section{Economic analysis}

The economic evaluation will be conducted in two ways. First we will conduct a cost-effectiveness analysis (CEA) with treatment response as the clinical outcome of interest. Second, we will conduct a Cost-Utility Analysis (CUA) using QALYs as a generic measure of health gains. Both CEA and CUA will be conducted from both the societal perspective (including indirect costs) and the health care perspective (direct healthcare costs). Analysis will be conducted using an intention to treat principle.

The Incremental Cost-Effectiveness Ratio (ICER) will be computed to obtain costs per treatment response and the costs per QALY gained. For decision-making purposes, the ICER acceptability curve will be plotted for various Willingness-To-Pay (WTP) ceilings, which helps 
to making judgements whether the intervention offers good value for money relative to CAU or no treatment.

\section{Trial status}

Enrolment for the trial started in January 2018. At the moment of submission of this manuscript, recruitment of participants is still open.

\section{Dissemination policy}

Trial results will be published as manuscripts in international peer reviewed journals and information bulletins to participants and personnel of participating dialysis centres. In close corporation with the Dutch Kidney Patient organisation we will disseminate the trial results among dialysis patients throughout the Netherlands. No professional writers will be used during the writing of the manuscripts. Authorship eligibility guidelines according to the international committee of medical journal editors (ICMJE) will be applied to all submitted manuscripts.

\section{Discussion}

There is a need for adequately powered RCT's to assess the effectiveness of treatment options for depressive symptoms in dialysis patients, as evidence is currently lacking. In order to conduct a high-quality trial, a multidisciplinary team with various experts related to dialysis and depression was involved in the development of the intervention and the study design. This trial may provide insights on the effectiveness, feasibility and applicability of Internet-based self-help PST in the dialysis population.

This study has several limitations. First, the intervention may not be applicable for patients with cognitive impairment, illiteracy, other cultural background or insufficient Dutch language skills. Second, Internet-based treatment on tablet-computers might not be suitable for dialysis patients who are not familiar with the Internet or tablet-use, or who suffer from vascular disease related problems such as impaired vision or polyneuropathy of their hands. Both of these problems will be solved by offering assistance by the study team during the intervention which will be documented, furthermore patients can be provided with a printed version of the intervention. Third, drop-out rates are high for internet-based self-help interventions in other populations [29, 30], to cope with this problem we embed the intervention in routine care in the dialysis departments during dialysis treatment with frequent face-to-face interaction with the participants, which will make it easier to prevent early termination. Furthermore, we aim to provide insight in the reasons for termination or non-participation in this study.
If demonstrated to be (cost) effective, Internet-based PST offers new possibilities to treat many dialysis patients with symptoms of depression and to improve the quality of care. This RCT is aimed at contributing to better recognition and adequate treatment of symptoms of depression in dialysis patients in the future.

\section{Supplementary information}

Supplementary information accompanies this paper at https://doi.org/10. 1186/s12888-019-2363-5.

Additional file 1. List of participating centres. Table of participating dialysis centres, corresponding cities and local investigators

\begin{abstract}
Abbreviations
BAI: Beck Anxiety Inventory; BDI-II: Beck Depression Inventory - second edition; BMI: Body Mass Index; CEA: Cost-Effectiveness Analysis; CUA: CostUtility Analysis; DSI: Dialysis Symptom Index; EQ-5D: 5 level EuroQol five dimension scale; ERA-EDTA: European Renal Association - European Dialysis and Transplant Association; ESRD: End Stage Renal Disease;

HPA: Hypothalamic-pituitary-adrenal; Hs-CRP: High sensitivity C-reactive protein; ICER: Incremental Cost-Effectiveness Ratio; IL-1B: Interleukin 1-beta; PST: problem solving therapy; QALY's: Quality-adjusted life years; QIDSSR16: Quick Inventory of Depressive Symptomatology; RCT: Randomized Controlled Trial; SF-12: Short Form 12; TNFa: Tumour necrosis factor alpha; WTP: Willingness-To-Pay
\end{abstract}

Acknowledgements

The authors are grateful to the participating dialysis centres and the trial team for their precious work.

\section{Authors' contributions}

All authors have contributed to study design and preparation of this manuscript. CEHS is the Principal Investigator for the DIVERS-II study and is grant holder. EN and RWS are implementing the trial. RWS designed the statistical analysis under supervision of FWD, CEHS and AH. EN is conducting the primary statistical analysis. EN, PO, CEHS and AH adjusted the intervention to dialysis patients. PO is responsible for the supported care within the intervention. EN and RWS wrote the first draft of this manuscript; all co-authors critically reviewed and revised the initial draft and approved the final version of the manuscript.

\section{Funding}

This trial is supported by ZonMW [grant number: 843001 804], OLVG and Stichting Zabawas. ZonMW has peer-reviewed and approved a previous version of this study protocol in the context of the grant application process, but had no role in data collection, data analysis and drafting or approving the present manuscript. ZonMW can be contacted via doelmatigheidsonderzoek@zonmw.nl.

\section{Availability of data and materials}

The final trial data set will be available only to the data analysts and not to the principal investigator, other members of the study team or local investigators. In the research contract signed by all participating centres is stated that OLVG will be owner of the final trial data set.

The datasets generated and/or analysed during the current study are not publicly available due to privacy reasons but will be available upon reasonable request from the principal investigator Dr. C.E.H. Siegert (e-mail: c. siegert@olvg.nl).

\section{Ethics approval and consent to participate}

The study protocol, information brochure and informed consent were approved by the Medical Ethics Committee of MEC-U, Nieuwegein, the Netherlands (registration number: NL58520.100.17) and covers all study sites. All potential participants will receive written and spoken information about the study as well as a reflection period until the next dialysis session before deciding to participate. Study participation is voluntary. Written informed consent will be obtained from all participants. 


\section{Consent for publication}

Not applicable.

\section{Competing interests}

The authors declare that they have no competing interests.

\section{Author details}

'Department of Nephrology, OLVG hospital, Jan Tooropstraat 164, 1061 AE Amsterdam, Netherlands. ${ }^{2}$ Department of Psychiatry, OLVG, Jan Tooropstraat 164, 1061 AE Amsterdam, Netherlands. ${ }^{3}$ Department of Amsterdam Public Health research institute, VUmc, Van der Boechorststraat 7, 1081 BT Amsterdam, Netherlands. ${ }^{4}$ Department of Clinical Epidemiology, Leiden University Medical Centre, Albinusdreef 2, 2333 ZA Leiden, Netherlands. ${ }^{5}$ Department of Psychiatry, Amsterdam University Medical Centre, Amsterdam and GGZ inGeest, Oldenaller 1, 1081 HJ Amsterdam, Netherlands.

Received: 18 September 2019 Accepted: 15 November 2019 Published online: 27 November 2019

\section{References}

1. Hedayati SS, Grambow SC, Szczech LA, Stechuchak KM, Allen AS Bosworth HB. Physician-diagnosed depression as a correlate of hospitalizations in patients receiving long-term hemodialysis. Am J Kidney Dis. 2005;46(4):642-9.

2. Palmer S, Vecchio M, Craig JC, Tonelli M, Johnson DW, Nicolucci A, et al. Prevalence of depression in chronic kidney disease: systematic review and meta-analysis of observational studies. Kidney Int. 2013;84(1):179-91.

3. Farrokhi F, Abedi N, Beyene J, Kurdyak P, Jassal SV. Association between depression and mortality in patients receiving long-term dialysis: a systematic review and meta-analysis. Am J Kidney Dis. 2014;63(4):623-35.

4. Lopes AA, Bragg J, Young E, Goodkin D, Mapes D, Combe C, et al. Depression as a predictor of mortality and hospitalization among hemodialysis patients in the United States and Europe. Kidney Int. 2002; 62(1):199-207.

5. Schouten RW, Haverkamp GL, Loosman WL, Chandie Shaw PK, van Ittersum FJ, Smets YFC, et al. Anxiety symptoms, mortality, and hospitalization in patients receiving maintenance Dialysis: a cohort study. Am J Kidney Dis. 2019;74(2):158-66.

6. Soykan A, Boztas H, Kutlay S, Ince E, Aygor B, Ozden A, et al. Depression and its 6-month course in untreated hemodialysis patients: a preliminary prospective follow-up study in Turkey. Int J Behav Med. 2004;11(4):243-6.

7. Palmer SC, Natale P, Ruospo M, Saglimbene VM, Rabindranath KS, Craig JC, et al. Antidepressants for treating depression in adults with end-stage kidney disease treated with dialysis. Cochrane Database Syst Rev. 2016;5: CD004541.

8. Friedli K, Guirguis A, Almond M, Day C, Chilcot J, Da Silva-Gane M, et al. Sertraline versus placebo in patients with major depressive disorder undergoing hemodialysis: a randomized, controlled feasibility trial. Clin J Am Soc Nephrol. 2017;12(2):280-6.

9. Winter SE, Barber JP. Should treatment for depression be based more on patient preference? Patient Prefer Adherence. 2013;7:1047-57.

10. Pena-Polanco JE, Mor MK, Tohme FA, Fine MJ, Palevsky PM, Weisbord SD. Acceptance of antidepressant treatment by patients on hemodialysis and their renal providers. Clin J Am Soc Nephrol. 2017;12(2):298-303.

11. Cukor D, Ver Halen N, Asher DR, Coplan JD, Weedon J, Wyka KE, et al. Psychosocial intervention improves depression, quality of life, and fluid adherence in hemodialysis. J Am Soc Nephrol. 2014;25(1):196-206.

12. Duarte PS, Miyazaki MC, Blay SL, Sesso R. Cognitive-behavioral group therapy is an effective treatment for major depression in hemodialysis patients. Kidney Int. 2009;76(4):414-21.

13. Kucuk $L$, Işil Ö. The Effects of Problem Solving Education on Depression Level and Problem Solving Skills on Dialysis Patientgft; 2009. p. 1638-49.

14. Gellatly J, Bower P, Hennessy S, Richards D, Gilbody S, Lovell K. What makes self-help interventions effective in the management of depressive symptoms? Meta-analysis and meta-regression. Psychol Med. 2007;37(9): 1217-28.

15. Beatty $L$, Lambert $S$. A systematic review of internet-based self-help therapeutic interventions to improve distress and disease-control among adults with chronic health conditions. Clin Psychol Rev. 2013;33(4):609-22.
16. Beltman MW, Voshaar RC, Speckens AE. Cognitive-behavioural therapy for depression in people with a somatic disease: meta-analysis of randomised controlled trials. Br J Psychiatry. 2010;197(1):11-9.

17. Dickens C, Cherrington A, Adeyemi I, Roughley K, Bower P, Garrett C, et al Characteristics of psychological interventions that improve depression in people with coronary heart disease: a systematic review and metaregression. Psychosom Med. 2013;75(2):211-21.

18. Ebert DD, Nobis S, Lehr D, Baumeister H, Riper H, Auerbach RP, et al. The 6month effectiveness of internet-based guided self-help for depression in adults with type 1 and 2 diabetes mellitus. Diabet Med. 2017;34(1):99-107.

19. Matcham F, Rayner L, Hutton J, Monk A, Steel C, Hotopf M. Self-help interventions for symptoms of depression, anxiety and psychological distress in patients with physical illnesses: a systematic review and metaanalysis. Clin Psychol Rev. 2014;34(2):141-57.

20. Andersson G, Cuijpers P, Carlbring P, Riper H, Hedman E. Guided internetbased vs. face-to-face cognitive behavior therapy for psychiatric and somatic disorders: a systematic review and meta-analysis. World Psychiatry. 2014;13(3):288-95.

21. Chan R, Dear BF, Titov N, Chow J, Suranyi M. Examining internet-delivered cognitive behaviour therapy for patients with chronic kidney disease on haemodialysis: a feasibility open trial. J Psychosom Res. 2016;89:78-84.

22. Erdley SD, Gellis ZD, Bogner HA, Kass DS, Green JA, Perkins RM. Problemsolving therapy to improve depression scores among older hemodialysis patients: a pilot randomized trial. Clin Nephrol. 2014;82(1):26-33.

23. Hernandez R, Burrows B, Wilund K, Cohn M, Xu S, Moskowitz JT. Feasibility of an internet-based positive psychological intervention for hemodialysis patients with symptoms of depression. Soc Work Health Care. 2018;57(10): 864-79.

24. Hudson JL, Moss-Morris R, Game D, Carroll A, Chilcot J. Improving distress in Dialysis (iDiD): a tailored CBT self-management treatment for patients undergoing dialysis. J Ren Care. 2016;42(4):223-38.

25. Warmerdam L, van Straten A, Twisk J, Riper H, Cuijpers P. Internet-based treatment for adults with depressive symptoms: randomized controlled trial. J Med Internet Res. 2008;10(4):e44.

26. Mynors-Wallis L. Problem solving treatment for anxiety and depression: a practical guide. New York: Oxford University Press; 2005.

27. Taraz M, Taraz S, Dashti-Khavidaki S. Association between depression and inflammatory/anti-inflammatory cytokines in chronic kidney disease and endstage renal disease patients: a review of literature. Hemodial Int. 2015;19(1):11-22.

28. Staufenbiel SM, Penninx BW, Spijker AT, Elzinga BM, van Rossum EF. Hair cortisol, stress exposure, and mental health in humans: a systematic review. Psychoneuroendocrinology. 2013;38(8):1220-35.

29. Boeschoten RE, Dekker J, Uitdehaag BM, Beekman AT, Hoogendoorn AW, Collette $\mathrm{EH}$, et al. Internet-based treatment for depression in multiple sclerosis: a randomized controlled trial. Mult Scler. 2017;23(8):1112-22.

30. van Straten A, Cuijpers P, Smits N. Effectiveness of a web-based self-help intervention for symptoms of depression, anxiety, and stress: randomized controlled trial. J Med Internet Res. 2008;10(1):e7.

31. Loosman WL, Siegert CE, Korzec A, Honig A. Validity of the hospital anxiety and depression scale and the Beck depression inventory for use in endstage renal disease patients. Br J Clin Psychol. 2010;49(Pt 4):507-16.

32. Council of Europe. Common European Framework of Reference for Languages: Learning, Teaching, Assessment (CEFR) 2018. Available from: https://www.coe.int/en/web/common-european-framework-referencelanguages/home.

33. Beck AT, Steer RA, Brown GK. The Beck depression inventory. Second edition ed. San Antonio: Psychological Corporation; 1996.

34. Beck AT, Steer RA, Brown GK. Does AJWvd. BDI-II manual: the Dutch version of the Beck depression inventory. 2nd ed. Enschede: Ipskamp; 2002.

35. Chilcot J, Wellsted D, Farrington K. Screening for depression while patients dialyse: an evaluation. Nephrol Dial Transpl. 2008;23(8):2653-9.

36. Rush AJ, Trivedi MH, Ibrahim HM, Carmody TJ, Arnow B, Klein DN, et al. The 16-item quick inventory of depressive symptomatology (QIDS), clinician rating (QIDS-C), and self-report (QIDS-SR): a psychometric evaluation in patients with chronic major depression. Biol Psychiatry. 2003;54(5):573-83.

37. Beck AT, Epstein N, Brown G, Steer RA. An inventory for measuring clinical anxiety: psychometric properties. J Consult Clin Psychol. 1988;56(6):893-7.

38. Loosman WL, Hoekstra T, van Dijk S, Terwee CB, Honig A, Siegert CE, et al. Short-form 12 or short-form 36 to measure quality-of-life changes in dialysis patients? Nephrol Dialysis Transpl. 2015;30(7):1170-6. 
39. Weisbord SD, Fried LF, Arnold RM, Rotondi AJ, Fine MJ, Levenson DJ, et al. Development of a symptom assessment instrument for chronic hemodialysis patients: the Dialysis symptom index. J Pain Symptom Manag. 2004:27(3):226-40.

40. Lamers LM, Stalmeier PF, McDonnell J, Krabbe PF, van Busschbach JJ. Measuring the quality of life in economic evaluations: the Dutch EQ-5D tariff. Ned Tijdschr Geneeskd. 2005;149(28):1574-8.

41. Bouwmans C, De Jong K, Timman R, Zijlstra-Vlasveld M, Van der FeltzCornelis C, Tan Swan S, et al. Feasibility, reliability and validity of a questionnaire on healthcare consumption and productivity loss in patients with a psychiatric disorder (TiC-P). BMC Health Serv Res. 2013;13:217.

42. Noppe G, de Rijke YB, Dorst K, van den Akker EL, van Rossum EF. LC-MS/ MS-based method for long-term steroid profiling in human scalp hair. Clin Endocrinol. 2015;83(2):162-6.

43. EDC C. Security Statement 2019. Available from: https://www.castoredc. com/security-statement/.

\section{Publisher's Note}

Springer Nature remains neutral with regard to jurisdictional claims in published maps and institutional affiliations.

Ready to submit your research? Choose BMC and benefit from:

- fast, convenient online submission

- thorough peer review by experienced researchers in your field

- rapid publication on acceptance

- support for research data, including large and complex data types

- gold Open Access which fosters wider collaboration and increased citations

- maximum visibility for your research: over $100 \mathrm{M}$ website views per year

At BMC, research is always in progress.

Learn more biomedcentral.com/submissions 